\title{
The Internal Cause and Formative Mech- anism of Enterprise Crisis in the View of Organizational Inertia
}

\author{
Chi Da \\ School of Public Administration and Law, Dalian University of Technology
}

\begin{abstract}
The uncertainty of external environment and the complexity of internal elements are making modern enterprises faced with more crises which makes crisis management for enterprises more difficult. So far, the attention paid to internal causes of enterprise crisis is not enough, neither is the research related to formative mechanism of enterprise crisis. This paper will put forward the origin of enterprises crisis based on dynamic unbalance of internal factors and external environment of enterprises. Then we will put forward the formative mechanism in the view of organizational inertia such as resource inertia, strategy inertia, structural inertia and cultural inertia.
\end{abstract}

Keywords: enterprise crisis; internal cause; organizational inertia; organizational mechanism

\section{Literature Review}

The study of crisis management started in the 1960s. It was between 1960s and 1980s that the theories of crisis management begun to spring up. The research field extended from political field to economic and social field. Crisis management has become a discipline, forming two independent branches, enterprises crisis management and public crisis man- agement. The representative figures for the former one are Barton, Foster, Green and Mitroff. ${ }^{[1]}$

So far, no agreements have been made about the connotation and the nature of enterprise crisis. Researchers of enterprise crisis usually regard "crisis" as a particular situation or condition. Barton stresses the "uncertainty" and the potential negative effects a crisis might bring to an organization ${ }^{[2]}$. While Hermann defines crisis as "a situation that threatens high priority goals of the decision-making unit, restricts the amount of time available for response before the decision is transformed and surprises the members of the decision-making unit by its occurrence" ${ }^{[3]}$. Mitroff holds that crisis is an actual threat to organizations. Bland regards crisis as an accident threatening the security, environment and credit of an enterprise $^{[4]}$. Booth defines crisis as a situation that can not be processed in normal procedure by any individual or organization $^{[5]}$. All those researchers focus on the abruptness of an accident, the lose control of condition and the urgency of situation. They tend to regard crisis as rare accidents. And they believe a crisis is random, unpredictable and disastrous to an organization.

Some researchers draw their attention to the relationship between the internal inertia of an organization and enterprise crisis, distinguishing the differences of two types of crisis and extending the under- 
standing of the nature of crisis. Hwang categorizes crisis into two types, "abrupt crisis" and "cumulative crisis". The abrupt crisis is the consequence of unpredictable external incidents. While the cumulative crisis is the result of accumulation of internal crisis factors ${ }^{[6]}$. As for the incentive of enterprise crisis, by observing internal factors such as the management mode, organizational structure and production technology, as well as external environment, Alves concludes that the internal factors could be controlled by managers.

Liang $^{[7]}$ suggests that an accidental disaster itself is not a crisis, but $r$ a trigger to a crisis. What internal mechanism can overcome is not a crisis for crisis is the problem within the mechanism. Whether the uncertain event will lead to crisis depends on the characteristics of an enterprise. He believes that "a crisis is the existing or potential factor threatening the existence and the development of an organization which shall not be solved without the effective organizational innovation. The nature of modern enterprise crisis is the defect of the institution within an enterprise. Li believes that the systematic entropy leads to the unbalance between the internal elements and the external environment which is the internal cause of enterprise crisis ${ }^{[8]}$. Zhang suggests that most of enterprise crisis can be seen as the result of the accumulation of unfavorable factors. The cause of enterprise crisis can be categorized as cognitive factor, organizational factor, communication factor and organizational environmental factor. Those views represent the development of enterprise crisis research.

\section{The Nature of Enterprise Crisis}

Psychologist Gerald Caplan points out that everyone is trying to keep inner steady and balanced with the environment.
While faced with severe challenge the balance is broken, it may lead to chaos in thoughts and behavior, which is the state of crisis. Caplan's theory shares commonness with enterprise crisis. Writer believes a crisis is the unbalance an enterprise faces, which can only be solved by reassessing behavior mode and cognitive mode.

The view that enterprise crisis is caused by both internal and external factors have been verified already. Shen and He studied cases of 28 enterprise which struggled in crisis and drew the conclusion that it was the accumulation of problems in organizational structure, institution, culture, strategy, resource, and technology that led to crisis ${ }^{[9]}$. In other words, the urgent events are the external behaviors of enterprise crisis. So it is wise to seek the root of crisis inside the enterprise.

The nature of enterprise crisis is the unbalance of "external environmentorganizational goal -- internal resource", which is the result of the internal organizational defects. Modern enterprises are faced with hyper-competitive environment. An enterprise needs dynamic capacity, a capacity to "integrate and rebuild internal and external to adjust to the environment" to establish dynamic balance between "environment - goal -- resource". So that an enterprise could adapt to the changing environment, avoid and solve crisis effectively. Teece suggests that a firm's competitive capacity is depended on the processes, asset position and evolution paths ${ }^{[10]}$. Integrating and rebuilding in organizational processes are not only evolving organizational routine but also absorbing environment information, reform cognitive mode. This process keeps a firm evolving its organizational mechanism which will ensure the dynamic balance of "environment - goal- resource" so as to avoid and coup with crisis effectively. In the changing environment, effective organizational mecha- 
nism consists of three parts, searching mechanism, selection mechanism and allocation mechanism ${ }^{[11]}$.

\subsection{Searching Mechanism}

The searching mechanism is the mechanism for searching, analyzing and explaining environmental information. It emphasizes a firm's sensitivity, cognitive ability to the dynamic environment, the goal's pertinence of a firm and the matching ability for environmental demand. It represents a firm's cognitive ability to external environment as well as the keen insight of a firm's strategy. The searching mechanism allows a firm to find out potential opportunities and threats in the changing environment, to analyze the importance and urgency of influence of environment, to resolve contradiction between environment and organization and to provide decision basis for managers.

\subsection{Selection Mechanism}

Based on the judgment of changing environment, the selection mechanism is established to reselect management modes, competition fields and even the goal for organization. How resources are distributed in a firm is depended on the goals and missions of the firm, while the goals and missions depend on the interactions of external market, internal resources and original path of a firm. The environment is complicated and the knowledge of manager is limited, the strategy will not always reach expected nor will it stay unchanged. If a firm held stubbornly on the same mode, crisis would be inevitable.

\subsection{Allocation Mechanism}

Allocation mechanism is the mechanism that a firm integrates and redistributes its resources to reply the environ- mental changes and to maintain competitive advantage. The change of resources allocation is implemented through the change in organizational structure, organizational routine and organizational norms. To maintain and promote competitive ability, a firm needs to recombine and redistribute resources to realize strategic objective. The function of resource allocation is to change and to enhance competitive ability through adjusting the direction resources are distributed and the way they are combined, so as to maintain the competitive ability in multiple fields. It will ensure the flexible development in various situations. In conclusion, in $\mathrm{dy}$ namic environment, the effective exertion of allocation mechanism is the basis for the dynamic balance of "environment goal -- resource".

\section{Organizational Inertia and Enter- prise Crisis}

The nature of enterprise crisis is the dynamic unbalance of "environment - goal -- resource", the root of which is the defects in organizational mechanism, the defects in searching mechanism, selection mechanism and allocation mechanism. Then what is the cause of these defects? This article will explain it in the view of organizational inertia. To avoid crisis effectively rely on the adjustment of internal factors. However, the organizational inertia hinders innovation within the organization. Organizational inertia is the internal cause of enterprise crisis.

Steiner points that the pattern external environment is more independent. The change in environment leads to unbalance between a firm and environment which is caused by organizational inertia. Organizations improve internal mechanism to avoid risk and crisis as a response to environmental changes. What is unchanged in the innovation is organizational inertia. 
No matter what the frequency of environmental changes is, organizations will still maintain the existed pattern, which is feature of organizational inertia. Gilbert categorizes organizational inertia into two types, resource rigidity and routine rigidity ${ }^{[12]}$. Resource rigidity is actually the sink cost brought by asset specificity. Routine rigidity is the inertia accumulated in organizational behavior mode, cognitive mode of a organization.

Therefore, we categorize organizational inertia into four types, resource inertia, strategy inertia, structure inertia, culture inertia. Together, they hinder the foundation and function of searching mechanism, selection mechanism and allocation mechanism in a firm, breaking the dynamic balance of "environment - goal -- resource".

\subsection{Resource Inertia and Enterprise Crisis}

Resource inertia is the unchangeable resource allocation mode, which is the result of asset specificity and sink cost. Asset specificity consists of site specificity, physical asset specificity and human specificity. The structure of existing asset is closely related to a firm's business field, combination of resources and product process. The self enhancement of the structure of existing asset will follow existing path, resource as specific asset will be transferred, and its value will drop. The sink cost produced by existing asset structure will delay the replacement of asset. When the external environment changes or product structure changes, existing asset will be hinder to innovation. Skills and knowledge could be regarded as technology and organizational specificity, once we transfer this asset to other fields, a large amount of transfer cost will be paid.

The organizational mechanism caused by resource inertia will firstly lead to barriers in allocation mechanism. Then it will limit a firm's searching and selecting behavior in dynamic environment, making it harder for a firm to change its field, breaking the dynamic balance of "environment - goal -- resource"

\subsection{Structure Inertia and Enterprise Crisis}

In general, structure concludes governance framework, management framework, and organizational framework. It is the steady arrangement of relationship of power and responsibility, operating rules and procedure. The result of Hannan and Freeman's research on organizational innovation shows that organization age, organization scale and the complexity of organization is negatively correlated with the speed of organizational innovation ${ }^{[13]}$. It means a film will be faced with more innovation resistance while it is growing.

The structure inertia is related with the history and scale of a firm. To begin with, there would be more rigid regulations. while a firm is growing, which enhances the structural inertia. Moreover, when a firm is expanding, there would be more levels in the hierarchy, and the relationship between departments becomes more sophisticated. Then a firm tends to repeat its behavior which slows down the speed to response external environmental changes.

\subsection{Strategy Inertia and Enterprise Crisis}

Strategy inertia refers to the framework that decision-makers response to environmental changes with action framework they get used to. The strategy inertia is formed in the past dynamic relationship of external environment and the internal resource. The knowledge and experience is accumulated and is repeated.

It presupposes the possible path and selection room for the strategy. When the external environment changes, strategy 
inertia prevents enterprise strategy to keep up with the environment.

Strategy inertia hinders enterprise to set up well-structured searching mechanism making a firm unable to make strategy response to the changing environment, breaking the dynamic balance of "environment - goal -- resource".

\subsection{Cultural Inertia and Enterprise Crisis}

Organizational culture is the common belief, basic cognitive style and behavior norms for all members in the organization. It is the steadiest element in an organization. It is also the most challenging part in organization innovation, which means it is an inertia hindering a firm to transfer its organizational mechanism.

Cultural inertia is presented as value preference and solidification cognitive mode. Cultural inertia affects individuals and groups by affecting potential value system, restraining cognitive mode and behavior. When environment changes, faced with severe external threats, a firm needs the support of organizational culture to provide them with basic measure of value. While the current culture tends to be laid back, stopping the firm from the innovation of cognitive mechanism, leading to the dynamic unbalance of "environment - goal -- resource".

\section{Conclusion}

Enterprise crisis is not steady and isolated. But rather it is systematic, dynamic and cumulative. As a consequence, we need to pay more attention to the internal factors of enterprise, so as to examine the relationship of external environment and internal factors. And it is wise to combine crisis management, strategy management and organizational innovation together. In this paper, we put forward enterprise crisis is the broken of dynamic balance of "environment - goal -- resource". The root of this unbalance is the defects of mechanism of a firm. While the defects of organizational mechanism is affects by organizational inertia. Hence, organizational inertia is the internal cause of enterprise crisis.

\section{References}

[1] C.W.Chen, "Enterprise Crisis and Crisis Management" Economic Forum, 1. 108-110,2009.

[2] L.Barton, Crisis in Organization: Managing and Communicating in the Heat of Chaos. 38, 1993.

[3] C.F.Hermann, Some consequences of crisis which limit the viability of organizations, Administrative Science Quarterly, 8. 1972.

[4] I. Mitroff, G.Anagnos. Managing Crises Before Happen, 2001.

[5] S.A.Booth, Crisis Management Strategy, 1993.

[6] P.Hwang, J.David. "Anatomy of Organizational Crisis". Journal of Contingencies and Crisis Management, 8(3).129-140, 2000

[7] J.H.Liang, C.H.Li, L.H.Gong, "Nature of Modern Enterprise Crisis" China Soft Science, 2, 89-94. 2004

[8] J.P.Li, C.Z.Zhu, S.Z.Li, "The Enterprise Crisis and Management Under the In-balance State," China Soft Science, 2, 47-50. 2002

[9] Z.L.Shen, J.D.He, “Types of Enterprise Crisis and Its Solution," Journal of Huazhong University of Science and Technology Edition of Social Sciences, 3, 83-88. 2002

[10] D.J.Teece, G.Pisano, A.Shuen. "Dynamic capabilities and strategic management," Strategic Management Journal, 18(7), 509-533. 1997 
[11] C.E.Helfat. Dynamic Capabilities:Understanding Strategic Change in Organizations. 2009.

[12] C.G.Gilbert, "Unbundling the structure of inertia: Resource versus routine rigidity," Academy of Management Journal, 48(5):741-763. 2005 